PROCEEDINGS OF THE WORLD CONFERENCE ON OZONE THERAPY IN MEDICINE, DENTISTRY AND VETERINARY. ANCONA (ITALY). SEPTEMBER 22nd - 23rd - 24th, 2017

\title{
Systemic Ozone Therapy as a complementary treatment in Internal Medicine [abstract]
}

\author{
Nicola Dardes \\ Guglielmo Marconi University, PAIDEIA Private Hospital, Rome, Italy
}

ABSTRACT

\section{OPEN ACCESS}

\section{Citation}

Dardes N. Systemic Ozone Therapy as a complementary treatment in Internal Medicine [abstract]. Proceedings of The World Conference on Ozone Therapy in Medicine, Dentistry and Veterinary. Ancona (Italy). September 22nd - 23rd - 24th , 2017. J Ozone Ther. 2019;3(4):37-40. doi: 10.7203/ jo3t.3.4.2019.15523

\section{Academic Editor \\ Jose Baeza-Noci, \\ School of Medicine, Valencia University, SPAIN}

\section{Editor}

World Federation of Ozone Therapy, Bolgna, ITALY

\section{Received}

June 17, 2019

\section{Accepted}

December 08, 2019

\section{Published}

December 30, 2019

\section{Intellectual Property}

Nicola Dardes.

This is an open access article distributed under the terms of the Creative Commons Attribution License (CC BY 4.0), which permits unrestricted use, distribution, and reproduction in any medium, provided the original author and source are credited.

\section{Author Information}

nicoladardes@hotmail.com
Introduction. Some data available in the literature and the common clinical experience of the physicians that use ozone therapy suggest that the treatment with systemic ozone is useful as supportive treatment in Internal Medicine. Nonetheless some criticism raise from the scientific community concerning the demonstration of the real effectiveness of this treatment. The criticism is based on the observation that the outcomes are often not clearly defined. Recently we published two studies in which the exercise capacity improved by systemic ozone both in patients with severe heart failure and in patients with COPD (Chronic Obstructive Pulmonary Disease) [1, 2].

Most of the patients admitted in the departments of Internal Medicine can be considered complex according to the Italian multicenter study (COMPLIMED - FADOI 2013). In a study recently published by our group similar results have been observed [3].

In clinical practice the therapeutic approach is aimed to treat each single, specific, pathophysiological target but in complex diseases a new approach should be desirable with the aim to support the effectiveness of the specific treatments in order to improve the clinical outcomes. To achieve this objective both clinicians and people involved in clinical research should take in account the scientific concept of complexity. According to its' definition a system can be defined as a complex system if it consist of many constituents interrelated which reciprocally interfere inducing a change of the equilibrium of the whole system. For this reasons the analysis of a complex system cannot be made by the evaluation of the single components but must be done by the evaluation of the global changes due to the interference of the different elements. It is well known that systemic administration of ozone induces changes in several physiological targets that contribute to obtain a re-modulation of the whole biological system. Therefore the systemic treatment with ozone could be considered of interest as a supportive treatment in internal medicine aimed to obtain a modulation of different pathophysiological targets in complex diseases.

Based on these clinical and theoretical assumptions different systematic observations have been carried out in our department in order to answer to the following questions about the efficacy and the usefulness of systemic ozone therapy in internal medicine:

1) May complexity be measured in Internal Medicine?

2) Does supportive systemic ozone therapy improve the outcome of the treatment in complex patients?

3) May oxidative stress value predict the effect of systemic ozone therapy in complex patients? 
According to the scientific and epistemological definition of complexity Morin and Prigogine (1996) stated that a complex system cannot be understood merely by the evaluation of the single constituents. By analogy the ultimate causes of a complex problem are not merely the bare bones. In fact a complex problem cannot be resolved simply by splitting up it in single factors but requires the analysis of the interaction between the constituent factors and a comprehensive vision.

In medical research and in clinical practice several factors must be considered in order to evaluate the complexity of a clinical condition:

1) the phenotype (the specific way to react to the pathogenic agent)

2) the specific pathophysiological changes due to the primary disease

3) the biological and biochemical features

4) the wear and the time course of the pathophysiological changes

5 ) the possibility to remove the primary cause of the disease

6) the associate pathologies

Materials and methods. As far as the measurement of complexity in medicine it concerns it is necessary to achieve at least three results:

1) to identify a multi-parametric function which could take in account the status variables involved

2) to homogenize different pathological condition on the basis of the changes of the whole alteration of the system

3) to evaluate the changes induced by treatments with multiple targets

According to these major premises a complexity score for clinical research has been built up by our group.

It has been named Paideia Complexity Score (PCS). PCS is divided in four indexes:

1) Quality Of Life Index from the Standard Questionnaire SF-36

2) Biological and Biochemical Index of Severity

3) Therapeutical Impact Index

4) Clinical Impression Index (C.I.)

Each index is graded in a numeric scale from 0 to 15 points. The maximum value of the score is 60 and the severity is graded into three groups: Mild (0-20); Moderate (20-40) ; Severe (40-60).

135 patients consecutively admitted in our department have been evaluated by the Paideia Complexity Score (PCS). The admittance diagnosis was: Chronic Obstructive Pulmonary Disease (59 pts); Ischemic Heart.

Disease (34 pts); Sleep Apnea Syndrome (29 pts); Diabetes Mellitus (13 pts). PCS resulted Mild or Moderate in 65 patients and Severe in 70 cases. The result indicates that more or less half of the patients admitted in a department of Internal Medicine must be considered in terms of complexity.

Effectiveness of Systemic Ozone Therapy in patients with moderate or severe complexity score (PCS) 70 pts with Severe PCS (>40) were treated by Systemic Ozone Therapy added to the regular medical treatment in order to evaluate the changes in the complexity score (PCS). Systemic Ozone Therapy was performed according to the standard procedure: ozone administered in $100 \mathrm{ml}$ of blood at a concentration ranging from $15 \mathrm{mcrg} / \mathrm{mL}$ to $25 \mathrm{mcrg} / \mathrm{mL}$ twice a week both during the hospitalization and after 
discharge from the hospital for six weeks. The changes in PCS were evaluated at the end of the treatment by a member of the medical staff unaware about the treatment with ozone (single blind). In 29 out of the 70 pts the PCS evaluated changed from severe to mild, in 7 patients a reduction from severe to moderate was recorded whereas 34 pts did not change the complexity score. 15 with PCS between 20 and 40 (moderate) who received the same schedule of treatment were also evaluated. In 13 pts the PCS changed from moderate to mild and in two pts no changes were recorded. No differences in terms of diagnosis and/or concomitant pathologies have been observed between the group of patients who improved the PCS and the group in which no changes were observed.

Oxidative stress and effectiveness of Systemic Ozone Therapy BAP (biological antioxidant potential) test and dROMs (reactive oxygen metabolites) test are commonly used as the laboratory tests aimed to measure the degree of the oxidative stress in several clinical conditions. Nonetheless systematic studies in different clinical conditions are not yet available in literature and the clinical usefulness of these test remains still unclear. On the other hand, in clinical practice, it is commonly accepted that high plasma levels of reactive oxygen metabolites indicate the presence of a condition of exposure to endogenous or exogenous oxidants both in healthy subjects and in patients. In order to evaluate the usefulness of the measurement of the plasma levels of the reactive oxygen metabolites in predicting the effectiveness of Systemic Ozone Therapy we evaluated:

1) The relationship between dROMs Values and PCS (Paideia Complexity Score) in 55 patients by simple linear regression.

2) The changes in dROMs in 21 COPD patients treated with Systemic Ozone Therapy.

3) The per cent changes of dROMs and of SF - 36 Quality of Life index in 25 patients with severe PCS (Paideia Complexity Score).

\section{Results.}

1) The correlation coefficient ( $r$ ) between dROMs and PCS resulted 34 . Therefore no correlation has been demonstrated between the level of oxidative stress and the degree of complexity of the pathologies treated.

2) COPD patients treated with Systemic Ozone Therapy showed an improvement of the dROMs values in 14 out of the 21 patients $(66 \%)$.

3) In 25 patients with severe PCS the treatment with Systemic Ozone Therapy induced a not significant decrease of $8 \%$ of the dROMs values whereas the Quality of Life SF - 36 increased of $40 \%$.

Conclusions. The systematic observation of patients admitted in our department of internal medicine indicate that roughly one half of cases must be considered characterized by the complexity. Therefore on theoretical basis the supportive treatment with Ozone should be considered useful. The use of an index of complexity allows to evaluate the effect of treatment with Systemic Ozone. According to the results obtained by the evaluation of the changes of the index of complexity the supportive treatment with ozone was effective in $58 \%$ of cases. No clinical and pathophysiological parameters have been identified to predict the effectiveness of the treatment. The evaluation of the oxidative stress do not correlate both with the degree of complexity of the disease and with the effectiveness of Systemic Ozone Therapy. 


\section{References}

1. Dardes N, et al. Ozone Therapy in Chronic Respiratory Failure. CHEST National Congress; 2015.

2. Dardes N, et al. Ozone as a complementary treatment in cardiovascular diseases. In: Integrative Cardiology. Springer International Publishing, Switzerland; 2017.

3. Dardes N, et al: The challenge of ozone therapy in Internal Medicine. In: Proceedings of the European Ozone Congress, Berlin; 2017. 